\title{
Correction of sun glint effect on MIVIS data of the Sicily campaign in July 2000
}

\author{
Rosa Maria Cavalli $\left({ }^{1}\right)$, Stefano Pignatti $\left({ }^{1}\right)\left({ }^{2}\right)$ and Elisabetta Zappitelli $\left({ }^{1}\right)$ \\ ( $\left.{ }^{1}\right)$ Laboratorio Aereo Ricerche Ambientali (LARA), IIA-CNR, Tor Vergata (RM), Italy \\ ${ }^{(2)}$ Istituto di Metodologie per l'Analisi Ambientale (IMAA), CNR, Tito Scalo (PZ), Italy
}

\begin{abstract}
To assess the suspended and dissolved matter in water in the visible and near infrared spectral regions it is necessary to estimate with adequate accuracy the water leaving radiance. Consequently radiance measured by a remote sensor has to be corrected from the atmospheric and the sea surface effects consisting in the path radiance and the sun and sky glitter radiance contributions. This paper describes the application of the sun glint correction scheme on to airborne hyperspectral MIVIS measurements acquired on the area of the Straits of Messina during the campaign in July 2000. In the Messina case study data have been corrected for the atmospheric effects and for the sun-glitter contribution evaluated following the method proposed by Cox and Munk (1954, 1956). Comparison between glitter contaminated and glitter free data has been made taking into account the radiance profiles relevant to selected scan lines and the spectra of different pixels belonging to the same scan line and located out and inside the sun glitter area. The results show that spectra after correction have the same profile as the contaminated ones, although, at this stage, free glint data have not yet been used in water constituent retrieval and consequently the reliability of such correction cannot be completely evaluated.
\end{abstract}

Key words MIVIS - water leaving radiance - sun glint - path radiance - probability density function

\section{Introduction}

The sea water color depends on the concentration of dissolved and suspended matter and on their scattering and absorption properties. Consequently the optical properties of the sea water constituents can be retrieved by means of airborne measurements of sea water radiance spectra. One of the major problems is the correction of the collected radiance from the atmospheric absorption and scattering and the effects due to the sunlight and skylight reflection

Mailing address: Dr. Rosa Maria Cavalli, Laboratorio Aereo Ricerche Ambientali (LARA), IIA-CNR, Via del Fosso del Cavaliere 100, 00133 Tor Vergata (RM), Italy; email: cavalli@lara.rm.cnr.it from the sea surface (Balducci et al., 1994). These contributions can lead to radiance errors, whose magnitude can exceed the signal from the water itself so as to lead to a much more difficult physically based retrieval of the sea parameters (Heege and Fisher, 2000). For remote sensing utilization, surface reflection of skylight can be calculated with sufficient accuracy with radiative transfer models, because it is basically independent of the sea state. Direct sunlight, on the contrary, shows strong spatial variations that require individual calculation and correction for each pixel depending on the sea state.

Non-correction of sun glint, the specular reflection from the direct sun radiance, leads to a restriction of reliable scenes according to geometric and sea conditions of exposure avoiding sun glitter.

The traditional model of sunglint basically convolves the Fresnel reflectivities assuming the slope distribution derived by Cox and Munk 
(1954, 1956). Many remote sensing retrieval algorithms also use the slope statistics of Cox and Munk to simulate the reflectance of the ocean surface while more recent slope measurements (like laser glitter, radar back scattering and microwave) have been used to define a small scale description of the sea surface roughness (Wenying et al., 2002).

The aim of this work is to apply the well known method proposed by Cox and Munk (1954, 1956) to MIVIS (Multispectral Infrared and Visible Imaging Spectrometer, Daedalus AA500) data acquired in the area of the Straits of Messina (fig. 1) to evaluate if an acceptable sun glint correction could be possible.

The MIVIS instrument is an airborne whisk broom scanner acquired by CNR (Italian National Research Council) in the framework of LARA (Airborne Laboratory for Environmental Studies). It is a simultaneous multispectral imaging system that operates in the range from visible to thermal-IR, with a high spectral resolution, 102 bands (table I), FoV (Field of View) and IFoV (Instantaneous Field of View) of $71.059^{\circ}$ and $2.0 \mathrm{mrad}$, respectively.

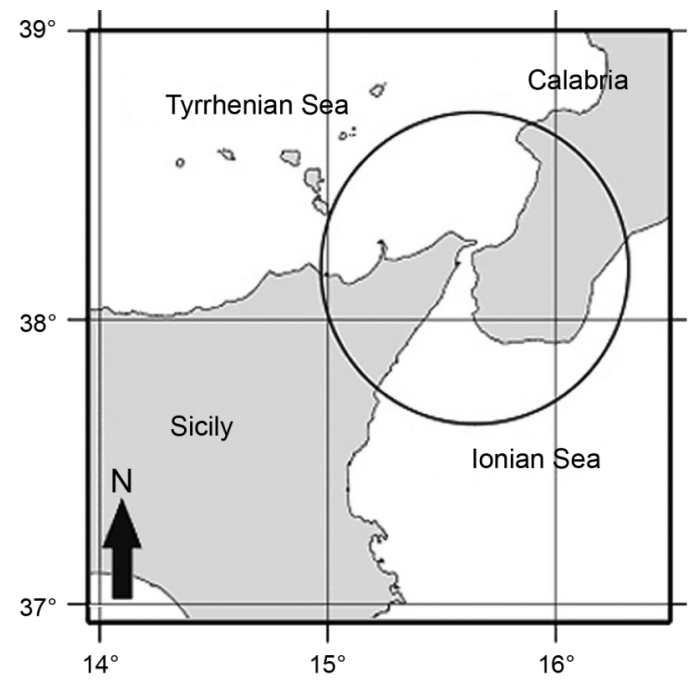

Fig. 1. The area investigated, the Straits of Messina (Southern Italy), identified by the circle in the figure.
Table I. MIVIS spectral characteristics.

\begin{tabular}{cccc}
\hline \hline Spectrometer & Bands & $\begin{array}{c}\text { Spectral } \\
\text { range [nm] }\end{array}$ & $\begin{array}{c}\text { Bandwidth } \\
{[\mathrm{nm}]}\end{array}$ \\
\hline 1 & $1-20$ & $430-830$ & 20 \\
2 & $21-28$ & $1150-1550$ & 50 \\
3 & $29-92$ & $1983-2478$ & 9 \\
4 & $93-102$ & $8180-12700$ & $340 \div 440$ \\
\hline
\end{tabular}

\section{Sunlint correction methodology}

To correct MIVIS images for atmospheric effects and sun glint contribution, a procedure has been developed taking into account the sea wave slope Probability Density Function (PDF) and modeling the water surface as a large number of small facets reflecting the sunlight (Cox and Munk, 1954, 1956)

The procedure has been developed in two processing steps. In the first one the sun glint contribution has been computed for pixels over sea in the MIVIS spectral range (440-830 nm) while in the second one the estimated sun glint radiance has been subtracted from the radiance corrected for atmospheric effects.

The instrument perceiving radiance $L$ in the spectral band centered at the wavelength $\lambda$, at height $h_{0}$, with sensor view angle $\theta$, can be considered as the sum of the following components (fig. 2):

$$
\begin{aligned}
& L\left(\lambda, h_{0}, \theta\right)=L_{\mathrm{P}}\left(\lambda, h_{0}, \theta\right)+L_{\mathrm{SKYG}}\left(\lambda, h_{0}, \theta\right)+ \\
& +L_{W C}\left(\lambda, h_{0}, \theta\right)+L_{\mathrm{SUNG}}\left(\lambda, h_{0}, \theta\right)+L_{\mathrm{W}}\left(\lambda, h_{0}, \theta\right)
\end{aligned}
$$

where $L_{\mathrm{P}}\left(\lambda, h_{0}, \theta\right)$ is the radiance generated along the optical path by atmospheric scattering (path radiance); $L_{\mathrm{SKYG}}\left(\lambda, h_{0}, \theta\right)$ is the radiance due to the specular reflection of atmospherically scattered light (sky glitter) by the sea surface; $L_{\mathrm{WC}}\left(\lambda, h_{0}, \theta\right)$ is the contribution due to sunlight and skylight reflection from whitecaps on the sea surface; $L_{\mathrm{SUNG}}\left(\lambda, h_{0}, \theta\right)$ is the radiance due to reflection of direct sunlight by the sea surface (sun glint); $L_{\mathrm{W}}\left(\lambda, h_{0}, \theta\right)$ is the water leaving radiance, the radiance emerging from the water and reaching the sensor. 


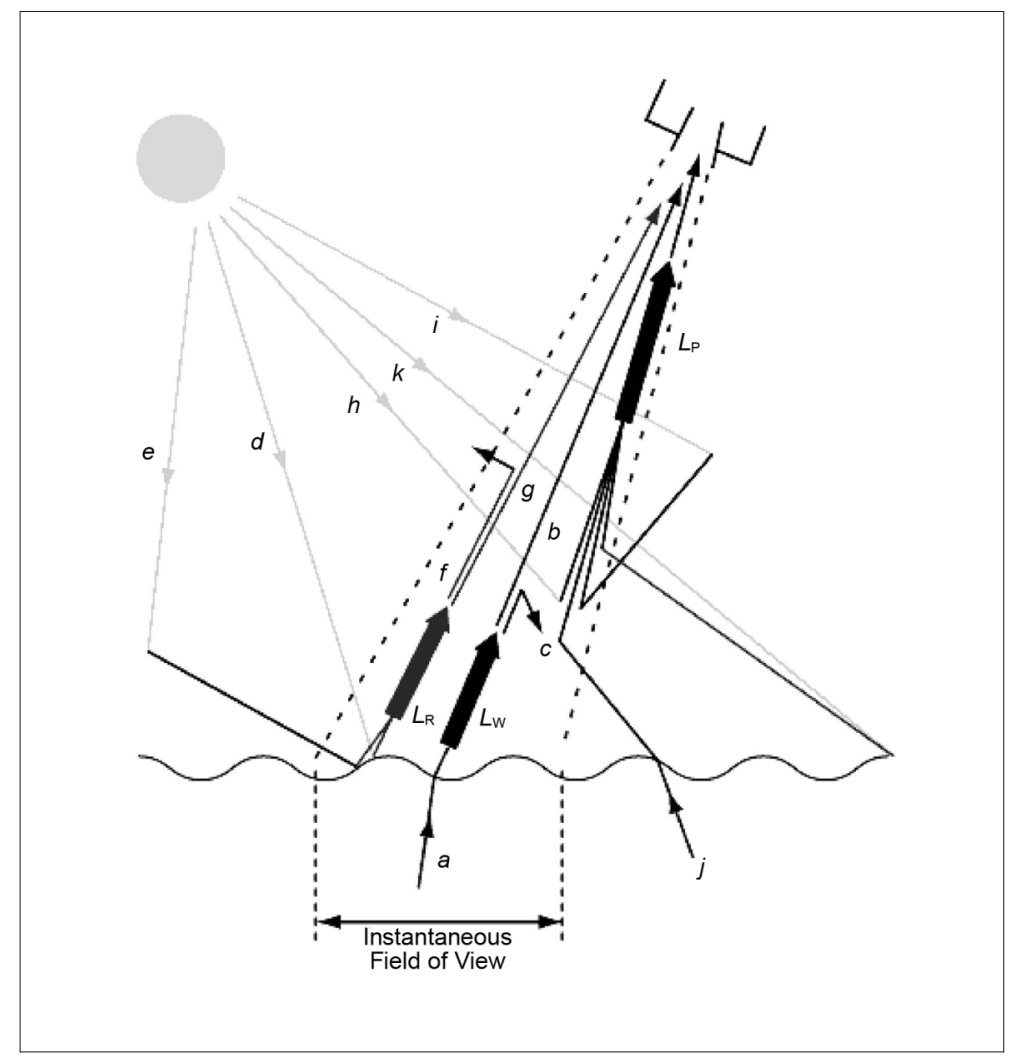

Fig. 2. Several different light pathways in the atmosphere are illustrated. $L_{\mathrm{W}}$ is the total water-leaving radiance. $L_{\mathrm{R}}$ is the radiance above the sea surface due to all surface reflection effects within the IFoV $\left(L_{\mathrm{SKYG}}, L_{\mathrm{WC}}\right.$ and $L_{\mathrm{SUNG}}$ ). $L_{\mathrm{P}}$ is the atmospheric path (www.physics.miami.edu).

The $L_{\mathrm{P}}$ component, just below the ozone layer, is given by the radiance contribution from multiple scattering by air molecules (Rayleigh scattering), multiple scattering by aerosols and the interaction between molecular and aerosol scattering (Gordon and Wang, 1994b). Its computation, depending on the local and seasonal conditions, has been performed by using MODTRAN v4 code Acharya (1998), configuring the input cards with MIVIS viewing geometry and the atmospheric characteristics during the flights.

The $L_{\text {SKYG }}$ radiance is predominantly independent on sea surface state when the sensor view angle is less than $60^{\circ}$ (Cox and Munk, 1956) and can be calculated with sufficient accuracy with radiative transfer models.
The term $L_{\mathrm{WC}}$ is related to the fractional whitecap area coverage often analyzed as a function of wind speed and investigated by Monahan (1971), Wu (1979), Monahan and Muircheartaigh (1980), Koepke (1984), Marks (1987), Gordon and Wang (1994a). Monahan (1971) expressed the dependence of oceanic whitecap coverage fraction $(F \mathrm{cov})$ on wind speed measured $10 \mathrm{~m}$ a.s.l. $(W)$ in the form

$$
F_{\mathrm{COV}}=a W^{\lambda}
$$

where, the least squares fitting method based on Monahan's data (1971) suggests for wind speed between $4 \mathrm{~ms}^{-1}$ and $10 \mathrm{~ms}^{-1}$ the values of

$$
a=1.35 \times 10^{-5} \text { and } \lambda=3.4 \text {. }
$$


Marks (1987) analyzed data collected during a cruise in the North Atlantic and Greenland Sea and the final result he obtained is given by the following relationship:

$$
F_{\mathrm{COV}}=2.54 \times 10^{-6} \mathrm{~W}^{3.58}
$$

with $W$ the wind speed at the standard $10 \mathrm{~m}$ above the sea surface.

It is generally accepted that whitecap coverage can be neglected for wind speeds less than $3 \mathrm{~ms}^{-1}$, while it becomes of particular importance for wind speeds above $9 \mathrm{~ms}^{-1}$. The corresponding whitecap reflectance contribution, related to the radiance term $L_{\mathrm{WC}}$, is small and can be estimated using

$$
\rho_{\mathrm{WC}}(\lambda)=6.49 \times 10^{-7} f_{\mathrm{wC}}(\lambda) \times W^{3.52} T\left(\theta_{0}, \lambda\right)
$$

where $f_{\mathrm{WC}}(\lambda)$ is a function expressing the spectral dependence of whitecap reflection, $W$ is the wind speed in $\mathrm{ms}^{-1}, T\left(\theta_{0}, \lambda\right)$ is the diffuse transmittance along the path from the sun to the ocean surface (Koepke, 1984; Gordon and Wang, 1994b). The spectral-dependence of the reflectance due to whitecaps is adopted from the work of Frouin et al. (1996).
The $L_{\text {SUNG }}$ term, according to Cox and Munk, can be retrieved by modeling the water surface as a large number of small facets which reflect the sunlight in all the directions including the specular one, the resulting radiance distribution showing the glitter patterns affecting the measurements. Cox and Munk took many photographs of sun glitter patterns on the ocean surface under different conditions while a nearby ship measured the wind speed and direction. Looking at a smooth ocean surface close to the specular angle, it would be possible to see a single spot, just a single reflection of the sun. However looking at a wind-roughened water surface, many small points of light can be observed. The glitter pattern, the collection of glitter points, carries information about the surface roughness. From these observations Cox and Munk derived an ocean wave slope PDF that can be described as a Gaussian curve plus higher-order skewness and kurtosis terms. The PDF data distribution fitted with a Gram-Charlier expansion can be expressed as

$$
p(x)=p_{0}(x)\left[1+\sum_{n=1}^{N} \frac{c_{n}}{n !} H_{n}(x)\right]
$$



Fig. 3. Sea-surface slope probability density function (solid line) shown with the best-fit Gaussian distribution (dashed line). Downwind slopes are positive. Zero is at nadir, indicating a horizontal surface. 
where $p_{0}(x)$ is the closest Gaussian to $p(x)$ and $H_{n}(x)$ are Hermite polynomials determine the coefficients $c_{n}$ that represent the deviation from the pure Gaussian case: the odd ones define the skewness term while the even ones the kurtosis term of the PDF, that is the degree of asymmetry and peaked ness of a distribution, respectively (fig. 3).

In the Cox and Munk model the sea surface is considered as a collection of facets, each with individual slope components $z_{x}$ and $z_{y}$ in the $x$ and $y$ direction. $p\left(z_{x}^{\prime}, z_{y}^{\prime}\right)$ is the resulting ocean wave slope PDF depending on the wind speed and direction

$$
\begin{aligned}
& p\left(z_{x}^{\prime}, z_{y}^{\prime}\right)=\left(\frac{1}{2 \pi \sigma_{c} \sigma_{u}}\right) \exp \left[-\frac{1}{2}\left(\xi^{2}+\eta^{2}\right)\right] . \\
& \left\{1-\frac{1}{2} c_{21}\left(\xi^{2}-1\right) \eta-\frac{1}{6} c_{03}\left(\eta^{3}-3 \eta\right)+\right. \\
& +\frac{1}{24} c_{40}\left(\xi^{4}-6 \xi^{2}+3\right)+\frac{1}{4} c_{22}\left(\xi^{2}-1\right)\left(\eta^{2}-1\right)+ \\
& \left.+\frac{1}{24} c_{04}\left(\eta^{4}-6 \eta^{2}+3\right)\right\}
\end{aligned}
$$

where $z_{x}^{\prime}$ and $z_{y}^{\prime}$ are function of $z_{x}$ and $z_{y}$, the crosswind and upwind components of the slope (fig. 4) given by

$$
z_{x}=\sin \alpha \tan \beta, z_{y}=\cos \alpha \tan \beta
$$

$\alpha$ is the facet azimuth angle and $\beta$ is the facet zenith angle. Let $\chi$ be the wind direction in the local frame ( $\chi$ taken clockwise from the sun) (fig. 4) and $W$ the wind speed modulus in $\mathrm{ms}^{-1}$. If the sun system $(x, y)$ is rotated through an angle $\chi$ to a new system $\left(x^{\prime}, y^{\prime}\right)$ related to the wind direction, then the facet slopes $z_{x}^{\prime}$ and $z_{y}^{\prime}$ in this wind system are

$$
\begin{gathered}
z_{x}^{\prime}=\sin \alpha^{\prime} \tan \beta, z_{y}^{\prime}=\cos \alpha^{\prime} \tan \beta \\
\alpha^{\prime}=\alpha-\chi
\end{gathered}
$$

therefore

$$
\begin{gathered}
z_{x}^{\prime}=\cos \chi \cdot z_{x}-\operatorname{sen} \chi \cdot z_{y} \\
z_{y}^{\prime}=-\operatorname{sen} \chi \cdot z_{x}+\cos \chi \cdot z_{y} .
\end{gathered}
$$

In eq. (2.3) $\sigma_{c}$ and $\sigma_{u}$ are the facet slope standard deviations in the crosswind $(c)$ and upwind $(u)$

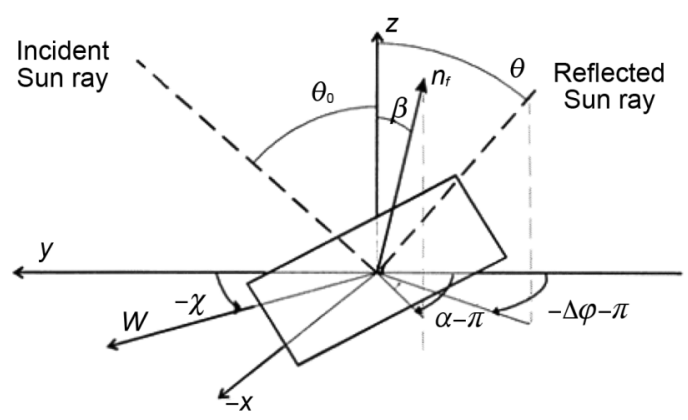

Fig. 4. Sun glint geometry: $\theta_{0}$ is the solar zenith angle, $\theta$ is the sensor zenith angle, $\Delta \varphi$ is difference between the solar and sensor azimuth angles, $\alpha$ and $\beta$ are the facet azimuth and zenith angle respectively, $W$ is the wind speed module and $\chi$ is the wind direction (Montagner and Billat, 2000).

$$
\begin{aligned}
& \sigma_{c}^{2}=0.003+1.92 \times 10^{-3} \mathrm{~W} \\
& \sigma_{u}^{2}=0.003+3.16 \times 10^{-3} \mathrm{~W}
\end{aligned}
$$

while $\xi$ and $\eta$ are the normalized $x$ and $y$ slopes defined as

$$
\xi=\frac{z_{x}^{\prime}}{\sigma_{c}}, \quad \eta=\frac{z_{y}^{\prime}}{\sigma_{u}}
$$

the skewness coefficients are

$$
\begin{aligned}
& c_{21}=0.001-0.0086 \mathrm{~W} \\
& c_{03}=0.004-0.0033 \mathrm{~W}
\end{aligned}
$$

and the kurtosis coefficients are

$$
c_{40}=0.40, c_{22}=0.12, c_{04}=0.23 \text {. }
$$

As a suitable first approximation of eq. (2.3) Cox and Munk used the isotropic Gaussian distribution to represent the oceanic wave slope PDF

$$
p\left(z_{x}, z_{y}\right)=\left(\frac{1}{\pi \sigma_{c m}^{2}}\right) \exp \left[-\frac{\left(z_{x}^{2}+z_{y}^{2}\right)}{\sigma_{c m}^{2}}\right] .
$$

By using this approximation the results can be simplified at the cost of omitting certain effects of wind directionality, for the mean square up/downwind slope component exceeds the cross-wind component and the up/downwind distribution is 

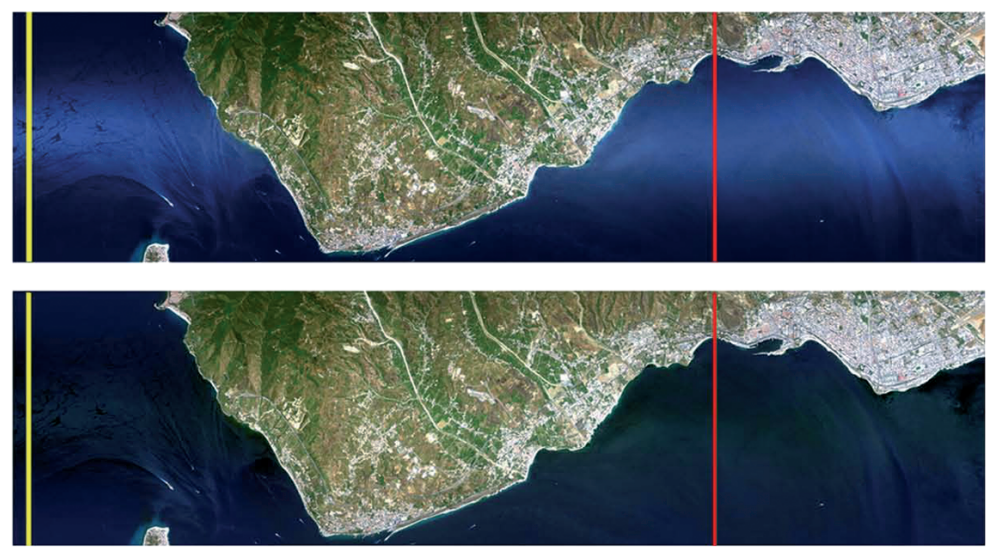

告
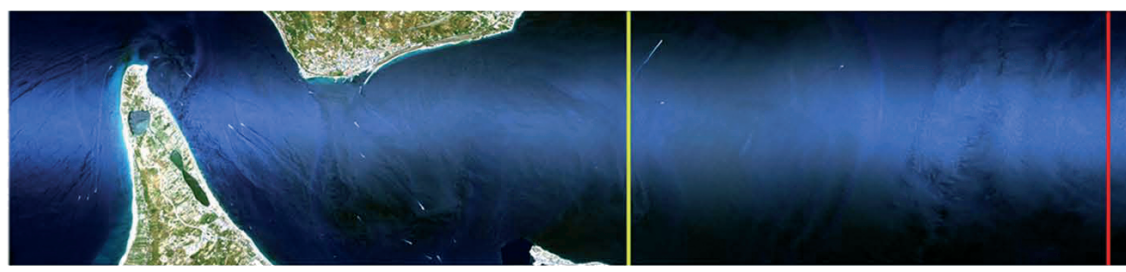

त

류

卷

正

๑ூ

纱 $\infty$

츨

$\sim$ 无
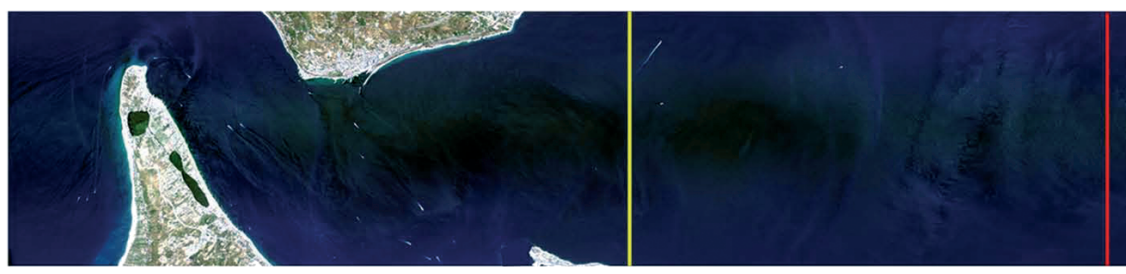

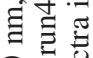

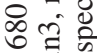

*

9 흠도

을

은

으릉

$\sum \approx$

$\sum i \frac{x}{0}$

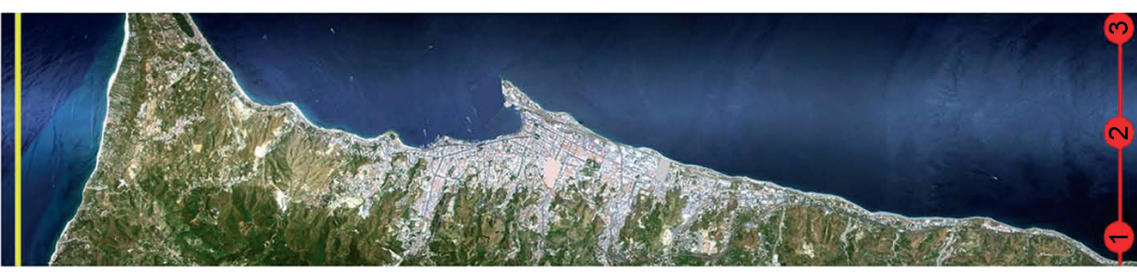

कै \&

일 을

ఏ 궝

ต บ

느음

ป气

巴ّ

Ð

恶产

ఏ छ



के



-

n.

ํㅜㄹ 
skewed while the mean square slope increases with the wind speed regardless of direction

$$
\sigma_{c m}^{2}=\sigma_{c}^{2}+\sigma_{u}^{2}=0.003+5.12 \times 10^{-3} W \text {. }
$$

According to eq. (2.4) the sun glint radiance $L_{\text {SUNG }}$ measured at $h_{0}$ is then given by

$$
\begin{aligned}
& L_{\mathrm{SUNG}}\left(\lambda, h_{0}, \theta\right)=E\left(\lambda, d, \theta_{0}\right) \cdot T_{D}\left(\lambda, \infty, \theta_{0}\right) \cdot \rho_{G} \cdot \\
& \cdot T_{U}\left(\lambda, h_{0}, \theta\right)
\end{aligned}
$$

where $E\left(\lambda, d, \theta_{0}\right)$ is the sun irradiance incident on the surface, function of $\lambda$ is the wavelength; $d$ is the sun-Earth distance at the time of measurement in Astronomical Units (mean sunEarth distance equal to $1 \mathrm{AU})$ and $\theta_{0}$ is the solar zenith angle; $T_{D}\left(\lambda, \infty, \theta_{0}\right)$ and $T_{U}\left(\lambda, h_{0}, \theta\right)$ are the downward and upward atmospheric transmittance, respectively; $\rho_{G}$ is the sun glint reflectance and is defined as

$$
\rho_{G}=\frac{\pi \cdot r(\omega)}{4 \cos \theta \cdot 4 \cos \theta_{0} \cdot \cos 4 \beta} \cdot p\left(z_{x}, z_{y}\right)
$$

$r(\omega)$ is the Fresnel reflection coefficient considered as a constant (0.02) for incidence less than $50^{\circ} ; \omega$ is the angle of incidence for a specular reflection between sun and receiver at a wave facet; $\theta$ is the sensor zenith angle; $\beta$ is the facet zenith angle.

\section{The case study of Messina}

The area investigated is the Straits of Messina (fig. 1), where the confluence of currents coming from the Tyrrhenian and Ionian Seas and the different characteristics of salinity and temperature of the two seas induce a particular oceanographic situation both from a biological and dynamical point of view (Magazzù et al., 1995).

Airborne data were acquired on July 2, 2000 starting at 10:43, by means of three flight runs (fig. 5), about $30 \mathrm{~km}$ length, flying at an altitude of $4500 \mathrm{~m}$ (corresponding to a nadir spatial resolution of $9 \mathrm{~m}$ ) and with a direction approximately S-N (table II) when a wind speed at $4 \mathrm{~ms}^{-1}$ during the airborne surveys was measured by the Agency for Air Navigation Services (ENAV) at the airport of Reggio Calabria. The airborne surveys were accompanied by a concomitant sea survey. Within the sea true campaign measurements optical sea water properties and water sampling at different depths for laboratory quantitative analysis were acquired.

The true color images depicted in fig. 5 show an evident sun glint reflection in the centre of the flight line according to the Snell Law with respect to the solar zenith angle. The effect of this reflection on the images strongly affects the data usefulness because in the glint area the spectral radiance behavior of the sea water is strongly modified. On the images it can be observed that in our case sun glint correction was unavoidable otherwise data would have been discarded, the measured radiance values being up to three times the corrected values. Therefore in order to remove the effects of the glint atmospheric contributions on the data the Cox and Munk correction method has been implemented to the images.

The correction of the sun glint atmospheric contributions on the images was taken into consideration by defining a procedure developed in the Interactive Data Language (IDL) language to be fully compatible with the MIVIS atmospheric and geometric pre-processing chain already developed in IDL.

The procedure is structured in three steps as shown in fig. 6 :

Table II. For each run the UTC, the solar zenith and sensor azimuth angles are shown.

\begin{tabular}{cccccc}
\hline \hline & UTC & Sensor height $(\mathrm{m})$ & Solar zenith angle & Solar azimuth angle & Sensor azimuth angle \\
\hline Run3 & $10: 43$ & 4500 & $15.63^{\circ}$ & $163.22^{\circ}$ & $13.00^{\circ}$ \\
Run4 & $11: 00$ & 4500 & $15.08^{\circ}$ & $177.86^{\circ}$ & $11.00^{\circ}$ \\
Run5 & $11: 16$ & 4500 & $15.35^{\circ}$ & $191.90^{\circ}$ & $14.00^{\circ}$ \\
\hline
\end{tabular}




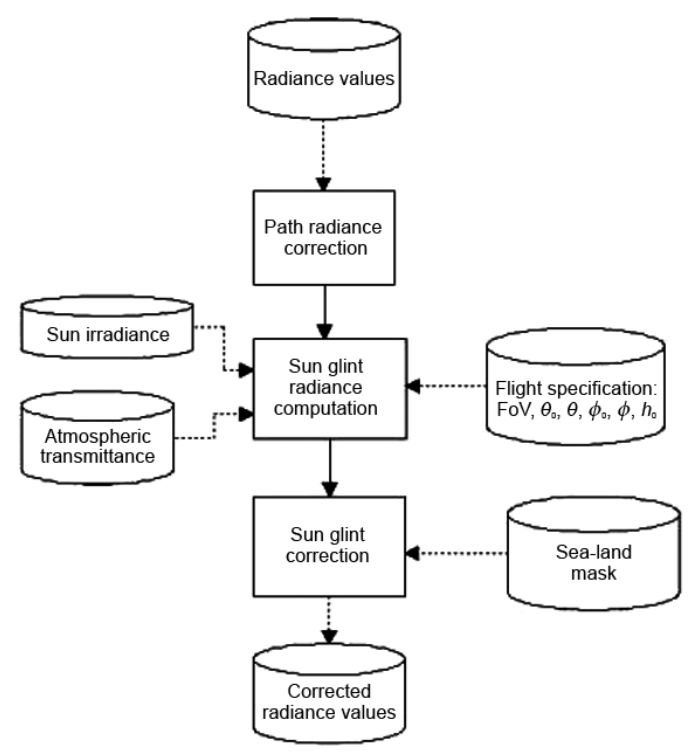

Fig. 6. Process chart of the sun glint correction.

i) Instrument perceiving radiance data of each flight run are corrected for atmospheric effects.

ii) The sun glint radiance is evaluated according to eq. (2.6) and considering the ocean wave slope probability density function in eq. (2.4).

iii) The sun glint contribution is then subtracted from the measured radiance already corrected for the atmospheric effects.

The path radiance, as well as the transmittance and the solar irradiance, were estimated by means of MODTRAN4 radiance simulations taking into account, the MIVIS optical characteristics (IFoV $2 \mathrm{mrad}, \mathrm{FoV} 71.2^{\circ}$, for a total of 755 pixels across track) the solar and observing geometry in table II, the 1976 US Standard atmosphere model, the ocean albedo and the maritime aerosol type. The profiles chosen for temperature pressure and molecular gases are the default ones for the selected atmospheric model.

For the case study of the Messina flights the contribution due to sunlight and skylight reflection from whitecaps on the sea surface was considered negligible in this case due to the low wind speed and the surface reflection of skylight was not computed. The resulting RGB images compared to the original ones are shown in fig. 5.

To evaluate the glint correction efficiency two different radiance profiles taken on the image at $560 \mathrm{~nm}$ relevant to two different scan lines of run3, run4 and run5 respectively (fig. 5) were selected. Radiance values before and after sun glint correction are compared showing the sun glint contamination removal in fig. 7. In all the three runs the computed Cox and Munk sun


Fig. 7. For each flight run two radiance profiles taken at $560 \mathrm{~nm}$ (the corresponding scan lines are traced in red and yellow in fig. 5) are displayed. Values before (black lines) and after correction (gray line) are compared. The applied slope probability density function is drown with a circle. 

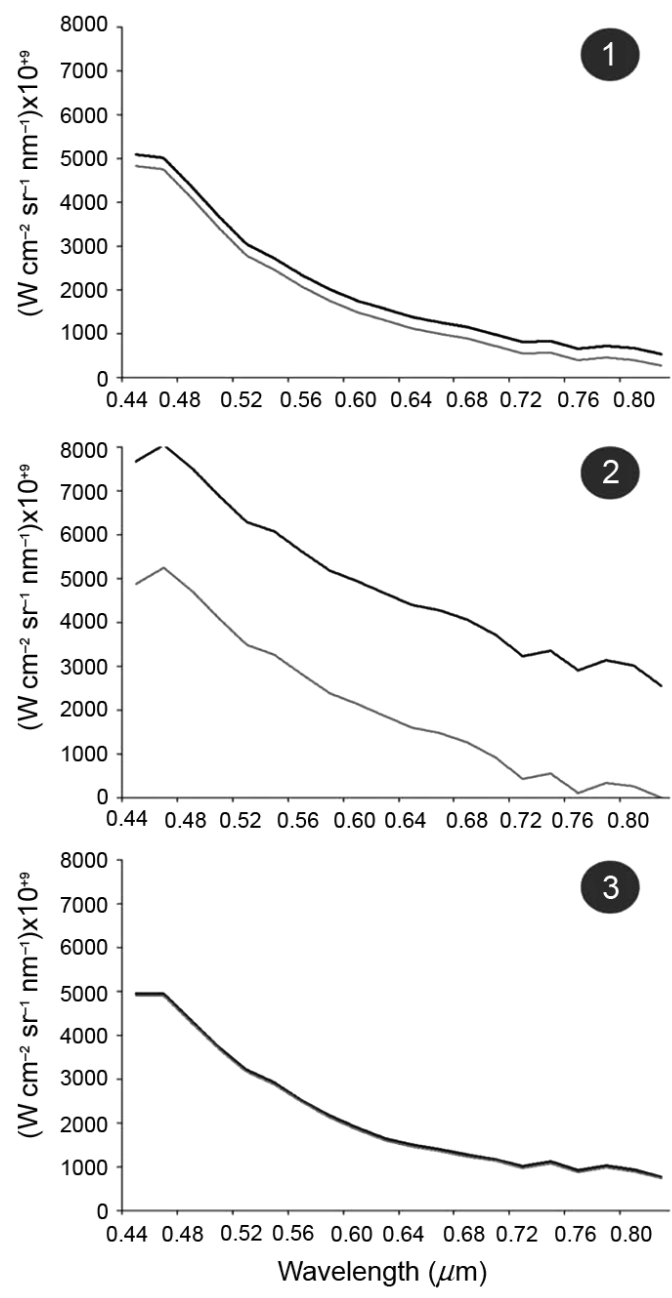

Fig. 8. Comparison between spectra before (in black) and after (in grey) the sun glint correction, relevant to three different pixels (showed in fig. 5 with point 1, 2 and 3 ) belonging to the same scan line (showed in red in fig. 5) selected in run3 and located out (point 1 and 3) and inside (point 2) the sun glitter area.

glint radiance (over-plotted with circle in fig. 7) well describes the measured radiance behavior with its maximum value placed is coincidence with the maximum of the sensor perceiving radiance. The peaks along the profile visible in all three runs could be related to local additive ef- fect of the radiance due to sea waves facet orientation that can locally determine a different sun glint reflection with respect to the Cox and Munk model average configuration applicable for the entire area.

A comparison between sun glitter contaminated and glitter free spectra, relevant to three different pixels belonging to the same scan line selected in run 3 and located out and inside the sun glitter area are shown in fig. 8. Spectra after correction show the same profile as the contaminated ones, although, at this stage of the work, free glint data have not yet be used in water inherent optical properties retrieval and consequently the reliability of the water spectral information cannot be wholly evaluated.

\section{Conclusions}

When performing the sun glint correction on airborne remotely sensed data on a coastline environment, some limitations have to be taken into account. The Cox and Munk model has been derived from observation of deep or open ocean water and, in the evaluation of the average sunglitter radiance, it assumes an isotropic distribution of the waves as only a function of wind speed and geometry. This approach is reasonable for satellite sensor with a wide spatial resolution, but may not be well suited when, as in this case, coastal waters and high spatial resolution are considered (Heege and Fisher, 2000). As a matter of fact, describing spatial small scale variations of sun glitters by means of a smooth probability density function could lead to an excessive correction with radiance values too low and/or the loss of typical spectral characteristics useful for water constituents retrieval (Guzzi et al., 1987). For an area like the Messina Straits, where the non linear morphology of the $\mathrm{E}$ and $\mathrm{W}$ coastline strongly controls the sea state, the configuration of the model with a uniform wind speed may not be sufficient, therefore swell, not related to local wind, should be taken into account. Also the interaction with the bathymetry is not considered, while the wave profile is distorted in shallow water areas even though in the Messina Straits the high depth gradient is present just close to the coastline. 
For the above mentioned reason the glint corrected spectra depicted in figs. 5 and 7 shows residual reflection on the images and peaks on the spectra that can be related to the presence of local sea states variation not taken into consideration in the model. Under the previous considerations the application of the Cox and Munk model to the case study of the Messina Straits has to be regarded as preliminary to following applications when data corrected for sun glint effects will be used in the computation of water constituents to test if the performed correction can be considered suitable. Therefore further application of this method on the area will require the estimation of the wind field (e.g., maps derived by active satellite) at a local state to take into consideration the local variation of the wave facets orientation at the time of the airborne surveys.

At this phase of the work this application of the method can be used, in any case, as a support to flag high values of sun glint once a threshold value is fixed. For MERIS (MEdium Resolution Imaging Spectrometer) satellite data, glint correction is not applied if sun glint reflectance exceeds a specified fraction of the observed reflectance to be defined ( 0.8 in MERIS case). In this case data should be flagged as contaminated glint (Montagner and Billat, 2000).

\section{REFERENCES}

Acharya, P.K., A. Berk, L.S. Bernstein, M.W. Matthew, S.M. Adler-Golden, D.C. Robertson, G.P. Anderson, J.H. Chetwynd, F.X. Kneizys, E.P. Shettle, L.W. Abreu, W.O. Galery, J.E.A. Selby and S.A. Clough (1998): MODTRAN User's Manual, Versions 3.7-4.0 (Air Force Research Laboratory, Space Vehicles Directorate, Air Force Materiel Command, Hanscom AFB, MA 01731-3010).

Balducci, A., P. Cipollini, G. Corsini and M. Diani (1994): A method for removing sea surface effects in airborne optical imagery, in Proceedings of the First Internation- al Airborne Remote Sensing Conference and Exihibition, 12-15 September, Strasbourg (France), Ann Arbor, MI: ERIMI, I, 131-140,

Cox, C. and W. Munk (1954): Measurements of the roughness of the sea surface from photographs of the sun's glitter, J. Opt. Soc. Am., 44 (11), 838-850.

Cox, C. and W. Munk (1956): Slopes of the sea surface deduced from photographs of the sun's glitter, Bull. Scripps Inst. Oceanogr., 6, 401-488.

Frouin, R., M. SchwindLing and P.Y. Deschamps (1996): Spectral reflectance of sea foam in the visible and nearinfrared: in situ measurements and remote sensing implications, J. Geophys. Res., 101, 14361-14371.

GorDON, H. R. and M. WANG (1994a): Influence of oceanic whitecaps on atmospheric correction of seaWiFS, Appl. Opt., 33, 7754-7763.

GordON, H.R. and M. WANG (1994b): Retrieval of waterleaving radiance and aerosol optical thickness over the oceans with SeaWiFS: a preliminary algorithm, Appl. Opt., 33, 443-452.

GuZZI, R., R. RiZZI and G. ZiBORDI (1987): Atmospheric correction of data measured by a flying platform over the sea: elements of a model and its experimental validation, Appl. Opt., 26 (15), 3043-3051.

HEEGE, T. and J. FisHeR (2000): Sun glitter correction in remote sensing imaging spectrometry, in SPIE Ocean Optics XV Conference, Monaco.

KoEPKE, P. (1984): Effective reflectance of oceanic whitecaps, Appl. Opt., 23, 1816-1824.

Magazzù, G., M. Aubert and F. Dicembrini (1995): The effect of tidal movements on planktonic transfer through the Straits of Messina, in Proceedings of the Symposium «The Straits of Messina Ecosystem», 4-6 Aprile 1991, Messina (Italy), 191-2002.

MARKS, R. (1987): Marine aerosols and whitecaps in the North Atlantic and Greenland Sea regions, Dt. Hydrogr. 40, 71-79.

Monahan, E.C. (1971): Oceanic white caps, J. Phys. Oceanogr., 1, 139-144.

Monahan, E.C. and I.O. Muircheartaigh (1980): Optimal power-law description of oceanic whitecap coverage dependance on wind speed, J. Phys. Oceanogr., 10, 2094-2099.

Montagner, F. and V. Billat (2000): Sun glint flag algorithm, MERIS Algorithm Theoretical Basis Documents (ATBD) 2.13, 4 (1), 13-1.

WeNYING, S., T.P. ChARLOCK and K. RutLEDGE (2002): Obesrvation of reflectance distribution around sunglint from a coastal ocean platform, Appl. Opt., 41 (35), 7360-7383.

Wu, J. (1979): Oceanic whitecaps and sea state, J. Phys. Oceanogr., 9, 1064-1068. 